\title{
Acoustic carpet cloak based on an ultrathin metasurface
}

\author{
Hussein Esfahlani, Sami Karkar, and Herve Lissek ${ }^{*}$ \\ Signal Processing Laboratory (LTS2), Ecole Polytechnique Fédérale de Lausanne, CH-1015 Lausanne, Switzerland \\ Juan R. Mosig \\ Laboratory of Electromagnetics and Antenna (LEMA), Ecole Polytechnique Fédérale de Lausanne, CH-1015 Lausanne, Switzerland
}

(Received 2 March 2016; revised manuscript received 19 May 2016; published 1 July 2016)

\begin{abstract}
An acoustic metasurface carpet cloak based on membrane-capped cavities is proposed and investigated numerically. This design has been chosen for allowing ultrathin geometries, although adapted to airborne sound frequencies in the range of $1 \mathrm{kHz}(\lambda \approx 30 \mathrm{~cm})$, surpassing the designs reported in the literature in terms of thinness. A formulation of generalized Snell's laws is first proposed, mapping the directions of the incident and reflected waves to the metasurface phase function. This relation is then applied to achieve a prescribed wavefront reflection direction, for a given incident direction, by controlling the acoustic impedance grading along the metasurface. The carpet cloak performance of the proposed acoustic metasurface is then assessed on a triangular bump obstacle, generally considered as a baseline configuration in the literature.
\end{abstract}

DOI: 10.1103/PhysRevB.94.014302

\section{INTRODUCTION}

Metasurfaces, first proposed in the electromagnetic realm, are artificially engineered surfaces composed of subwavelength unit cells which manipulate the reflected and (or) transmitted wavefronts in a preferred, predefined, and unconventional way. The peculiar macroscopic behavior of metasurfaces in response to incident waves led to the revisitation of the laws of reflection and refraction, and the generalized Snell's law was proposed to deal with such surfaces [1]. In addition to the wavefront manipulation [2], the functionality of these surfaces can be extended to wave absorption engineering [3], polarization tailoring [4], frequency filtering [5], and metasurface carpet cloaking [6-9].

Recently, the concept of acoustic metasurface has been proposed to manipulate the reflected or transmitted wavefronts of the impinging acoustic wave [10] and the functionality of these surfaces has been extended to acoustic matching [11], unidirectional transmission [12,13], lensing [14], absorbers [15,16], and cloaking [17,18].

Shaping wavefronts using an acoustic metasurface requires one to control the phase of the transmitted/reflected wave. In several studies on acoustic metasurfaces [19-22] the phase control has been achieved using a unit cell presenting a space coiling structure [23]. Space coiling is a technique where the phase control is done by the accumulation of the phase along the sound path in a labyrinthine-type structure [24]. Thus, the acoustic wave travels an effective acoustic path to ensure a certain phase lag/lead (compared to a predefined reference phase) to the price of relatively bulky unit cells $[25,26]$. Another technique is to use various materials with different refractive indices but similar acoustic impedances [27].

\footnotetext{
*herve.lissek@epfl.ch

Published by the American Physical Society under the terms of the Creative Commons Attribution 3.0 License. Further distribution of this work must maintain attribution to the author(s) and the published article's title, journal citation, and DOI.
}

Recently, Helmholtz resonators [28-30] and membrane [32] and pentamode metamaterial [33] based unit cells have been proposed as acoustic metasurface unit-cell designs to control the phase of the acoustic wave. However, most of these studies on metasurface unit-cell designs still suffer from being bulky, having limited bandwidth, high losses, and nonuniform reflected/transmitted wave amplitude and do not present the aptitude for reconfigurability.

According to the impedance-governed generalized Snell's law of reflection [34], the acoustic impedance of the surface can be envisaged as the controlling factor to engineer the reflection phase. Thus, using a surface impedance strategy, a unit-cell design is proposed, aiming at saving space and providing much thinner geometries than the ones reported so far. To achieve the desired thin acoustic metasurface, lumped element acoustic modules representing acoustic impedances should be proposed with an easily tunable mass/compliance (inductive/capacitive) behavior.

In this paper, a new type of unit cell is proposed, owing to an acoustic impedance-based design, for use in a reflector-type acoustic metasurface. The phase of the reflected wave for such a unit cell is controlled based on the inductive and capacitive nature of constituent elements. The proposed unit cell is composed of a hard wall cavity which is capped with a membrane clamped at the edges. The cavity contributes to the compliance (capacitive) part of the total acoustic impedance while the clamped membrane presents compliance/mass (capacitive/inductive) behavior in response to the impinging acoustic wave [35]. Since the impedance of a clamped membrane is a function of the geometrical dimensions and material properties, it can be easily modified, e.g., by varying the thickness of the membrane [36]. Thereby, the total surface acoustic impedance of the unit cell can be easily tuned to desired values. The proposed ultrathin unit cell $(\lambda / 30)$ is deployed within a reflectarray-type metasurface allowing the manipulation of reflected wavefronts. The introduced flat surface may be used to replace a curved reflector as a secondary antenna or be used for deflecting or concentrating sound in a point. Finally, it is shown that the functionalities of acoustic metasurfaces can be extended to acoustic carpet 


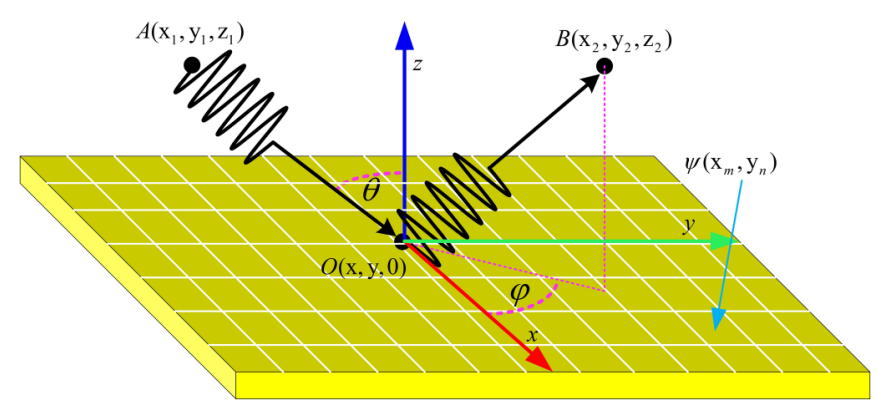

FIG. 1. Acoustic wave impinges a surface with nonzero phase gradient.

cloaking, exactly like in the electromagnetic counterpart. The proposed surface impedance based unit cell is sufficient to design skinlike cloaks and create the illusion of bare ground when an object is covered by such a metasurface based cloak.

\section{REFLECTOR-TYPE ACOUSTIC METASURFACE}

Acoustic reflectarray metasurfaces, aiming at manipulating the reflected wavefronts, do not obey Snell's law at the macroscopic level. To mathematically describe these surfaces, Snell's law should be revisited. In optics this modification has been done using Fermat's principle under the name of generalized laws of reflection and refraction [1], while in acoustics it was treated using Green's function under the name of the impedance-governed generalized Snell's law [10]. Here, Fermat's principle is used to derive the generalized Snell's law for acoustic reflector-type metasurfaces.

\section{A. Fermat's principle}

Let us suppose that a monochromatic acoustic plane wave, propagating in a fluid medium, impinges a lossless inhomogeneous surface with subwavelength reflection phase discontinuity. According to Fermat's principle, the path traveled between two points $A$ and $B$ by the sound wave is the path that can be traversed in the least time. The mathematical interpretation of Fermat's principle, illustrated in Fig. 1, is

$$
\tau_{\text {total }}=\frac{O A+O B}{c}+\frac{\psi(x, y)}{\omega_{0}}
$$

where $A, O$, and $B$ are the source location, intersection between the ray and the surface, and observation point, respectively; $c$ is the speed of sound in the fluid domain; and $\psi(x, y)$ is the surface phase function (phase of the reflection coefficient). Fermat's principle dictates that the effective trajectory followed by the wave between two fixed points, $A$ and $B$, should be minimal. This can be mathematically interpreted as finding the critical points of Eq. (1) based on $\frac{\partial \tau_{\text {total }}}{\partial x}=0$ and $\frac{\partial \tau_{\text {total }}}{\partial y}=0$. After simplification, Fermat's principle is summarized as a generalized Snell's law for acoustic waves:

$$
\begin{aligned}
\sin \theta_{r} \cos \phi_{r}+\sin \theta_{i} \cos \phi_{i} & =-\frac{1}{k} \frac{\partial \psi(x, y)}{\partial x}, \\
\sin \theta_{r} \sin \phi_{r}+\sin \theta_{i} \sin \phi_{i} & =-\frac{1}{k} \frac{\partial \psi(x, y)}{\partial y}
\end{aligned}
$$

where $\phi$ is the azimuth angle with respect to $x$-axis, $\theta$ is the elevation angle with respect to $z$-axis, $k$ is the wave number and the subscript $i$ and $r$ are indicating incident and reflected waves, respectively. Eq. (2) reveals that the gradient of the phase over the surface determines the reflected wave direction.

\section{B. Acoustic impedance and generalized Snell's law}

Let us discretize the surface of Fig. 1 into subwavelength rectangles of $d_{x} \times d_{y}$ in the $x y$ plane, denoted as unit cells. Equation (2) can be reformulated and presented as a single equation which describes the discretized phase function over the surface for predefined incident and reflected wave angles:

$$
\begin{aligned}
\psi\left(x_{m}, y_{n}\right)= & -k d_{x} m\left(\sin \theta_{r} \cos \phi_{r}+\sin \theta_{i} \cos \phi_{i}\right) \\
& -k d_{y} n\left(\sin \theta_{r} \sin \phi_{r}+\sin \theta_{i} \sin \phi_{i}\right)
\end{aligned}
$$

where $m$ and $n$ are integers and represent the indices of the discrete unit cells along $x$ and $y$, respectively. Considering $\psi$ as a surface phase function to achieve predefined reflection angle $\left(\theta_{r}, \phi_{r}\right)$, the phase of the surface reflection coefficient should be engineered accordingly.

Since the reflection coefficient of the surface is governed by its acoustic impedance, the phase function of the metasurface can be attributed to the acoustic impedance of the surface. Thus, by properly choosing the acoustic impedance of each unit cell on the surface, a metasurface with anomalous reflection behavior can be designed. In order to realize a nonzero phase gradient surface to fully control the reflected wave, a unit-cell configuration which can produce a full phase range $(0-2 \pi)$ reflection coefficient is proposed in the next section, where the acoustic impedance is easily tuned by slight changes in the geometry.

\section{METASURFACE UNIT CELL}

To design a unit cell which covers the full phase range for reflected waves, an acoustic configuration with both mass/compliance behavior (inductive/capacitive nature) is required. The most straightforward acoustic module with an inductive/capacitive reactive part is a Helmholtz resonator, where the cavity presents compliant behavior and the narrow neck represents the mass [28-31]. However, Helmholtz resonators are relativity bulky modules and their acoustic behavior highly depends on their dimensions. Hence Helmholtz resonators are not good candidates for ultrathin metasurfaces.

\section{A. Analytical modeling}

The proposed metasurface unit cell is composed of an air-filled cubic cavity with acoustically hard walls and a vibrating membrane which is clamped at its edges to the cavity, in which the damping will be first neglected (the effects of damping will be further discussed in Sec. V B). The cavity with subwavelength dimensions can be modeled as an acoustic compliance (capacitance):

$$
C_{a b}=\frac{V_{b}}{\rho_{\text {air }} c_{\text {air }}^{2}}
$$

where $V_{b}$ is the volume of the cavity and $\left(\rho_{\text {air }}, c_{\text {air }}\right)$ are the mass density and sound velocity of the air, respectively. The membrane contributes to both mass/compliance 

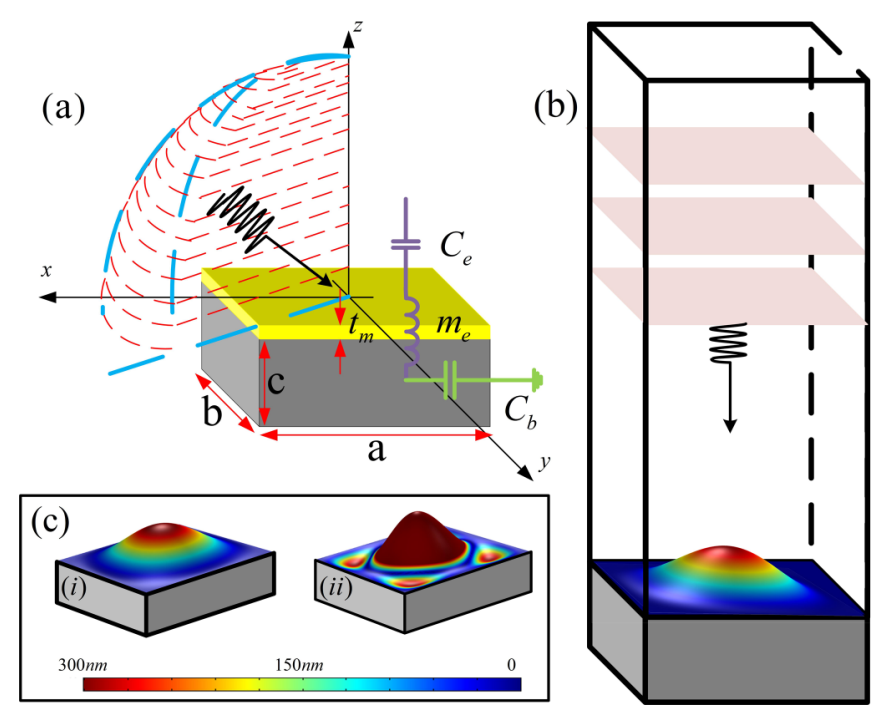

FIG. 2. (a) Acoustic metasurface unit cell and corresponding
ansmission-line model. The unit cell is composed of a cavity (gray) with acoustically hard walls which is capped with a clamped membrane (yellow). The reflection phase study zone is shown in red dashed lines. (b) To retrieve the phase profile of the unit cell, it is placed in a waveguide simulator. (c) Eigenmodes of the unit cell for high and low thickness membranes (i) $t_{m}=266 \mu \mathrm{m}$ and (ii) $t_{m}=86 \mu \mathrm{m}$.

(inductive/capacitive) behavior depending on its geometrical dimensions, material properties, and operating frequency. The lossless clamped rectangular membrane vibrating on its first mode can be modeled with the lumped element circuit model of Fig. 2(a), the values of equivalent acoustic mass $m_{a e}$ and compliance $C_{a e}$ being given by

$$
\begin{aligned}
C_{a e} & =\frac{1}{(a b)^{2}}\left(\frac{a^{3}\left(1-v^{2}\right)}{\pi^{4} E t_{m}^{3} b\left[1+\frac{2}{3}(1-v)\left(\frac{a}{b}\right)^{2}+\left(\frac{a}{b}\right)^{4}\right]}\right) \\
m_{a e} & =\frac{1}{(a b)^{2}} \frac{9}{4} \rho_{m} t_{m} a b
\end{aligned}
$$

where $\left(a, b, t_{m}\right)$ represent the membrane length, width, and thickness and $\left(\rho_{m}, E, v\right)$ are the mass density, the bulk modulus, and the Poisson ratio of the membrane, respectively (see the Appendix).

Using the transmission-line model of Fig. 2(a), the total acoustic impedance of the proposed unit cell $Z_{a t}$ is calculated by

$$
Z_{a t}=j \omega m_{a e}+\frac{1}{j \omega C_{a e}}+\frac{1}{j \omega C_{a b}} .
$$

This analytical model of the unit-cell acoustic impedance is illustrated in Fig. 3 and compared to a numerical model achieved with finite element software (Comsol Multi-Physics), further described in the following. It shows that the analytical model of $Z_{a t}$ qualitatively follows the numerical results for thick membranes. However, the model tends to diverge from numerical results for unit cells with thinner membranes. This is due to the excitation of higher-order modes for unit cells with thin membranes. The unit-cell membrane vibrates in its first mode [Fig. 2(c-i)] for the thickness $>120 \mu \mathrm{m}$ as shown in an eigenfrequency study in the inset of Fig. 3 and this

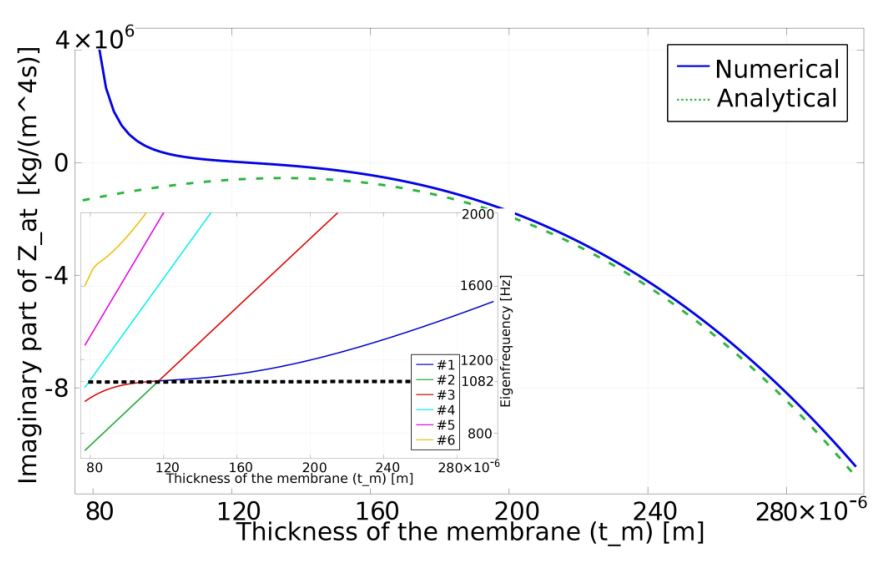

FIG. 3. Imaginary part of the acoustic impedance of the unit cell at $f=1082 \mathrm{~Hz}$ : numerical vs analytical method. (Inset) First six eigensolutions of the unit cell vs thickness of the membrane. The unitcell dimensions are $23 \times 23 \times 10 \mathrm{~mm}$ capped by a Kapton membrane.

is consistent with the first mode excitation assumption to derive Eq. (5). However, the assumption is violated for thin membranes $(<120 \mu \mathrm{m})$ as shown in Fig. 2(c-ii). Moreover, the dependency of the unit-cell impedance to the incident angle is neglected in the analytical model of $Z_{a t}$. Considering the angular dependency of the total impedance, the reflection coefficient of the unit cell is found to be

$$
\Gamma\left(\theta_{i}, \phi_{i}\right)=\frac{Z_{a t}\left(\theta_{i}, \phi_{i}\right)-Z_{a c}}{Z_{a t}\left(\theta_{i}, \phi_{i}\right)+Z_{a c}}
$$

where $Z_{a c}=\frac{\rho_{\text {air }} c_{\text {air }}}{a b}$ is the acoustic characteristic impedance of the medium in the waveguide of section $a b$ and $Z_{a t}\left(\theta_{i}, \phi_{i}\right)$ is the direction dependent total acoustical impedance of the unit cell.

A close study of Eqs. (4)-(7) reveals that the phase of the reflection coefficient is a function of geometrical dimensions and material properties of the unit cell, operating frequency, and direction of wave incidence. Moreover, encapsulated in Eq. (5), the inductive/capacitive nature of the membrane highly depends on its thickness $\left(t_{m}\right)$. Thus, the phase of the reflection coefficient can sweep the full phase range by varying the thickness of the membrane in the range of a few hundreds of micrometers. Using the proposed configuration, the designed unit cells of almost similar geometrical dimensions but different impedances can cover a surface of any shape, like LEGO pieces. Moreover, by replacing the passive membranes with active ones, tunable unit cells can be designed [37].

Although the presented analytical modeling provides a qualitative description of the membrane capped unit cell, as discussed it is only valid for the first vibrational mode of the membrane in the normal incident condition. A full wave simulation of the structure should be performed to retrieve the unit cell accurate reflection coefficient values, taking into account the incident direction and the higher-order modes.

\section{B. Numerical simulation}

The above-mentioned finite element software (Comsol Multi-Physics) is used for the numerical simulations of the unit cell. An acoustic-shell interaction physics module is used to extract the phase of the reflection coefficient. The unit-cell cavity is set to "sound hard boundary conditions" and the 
membrane is assumed to be clamped at four edges to the cavity. The simulation geometry is completed by placing the unit cell within a waveguide of rectangular section $a b$ [see Fig. 2(b)]. In order to consider effects of adjacent unit cells (mutual coupling) for the case of slant incident waves, the boundaries of the waveguide simulator are set to "Floquet boundary condition" while the radiation boundary condition is assigned to the upper extremity of the waveguide. The dependence of the unit cell $Z_{a t}\left(\theta_{i}, \phi_{i}\right)$ to the direction of the incident wave is also accounted for by sweeping it in both the azimuth and elevation plane. For a square cavity $(a=b)$ there are four symmetry lines [Fig. 2(a)] and the reflection phase study zone is reduced to $\left(0<\phi<\frac{\pi}{4}, 0<\theta<\frac{\pi}{2}\right)$. The reflection coefficient is retrieved using a two-microphone measurement method [38].

The unit-cell dimensions are designed to be $23 \times 23 \times 10 \mathrm{~mm}$ capped by a Kapton membrane with the following material properties: $E=2.758 \mathrm{GPa}, v=0.34$, and $\rho_{m}=1420 \mathrm{~kg} / \mathrm{m}^{3}$. The membrane is considered lossless for the time being, the effects of losses being assessed in Sec. V B. The operating frequency is set to $f=1082 \mathrm{~Hz}$, and the fluid medium is air with $c_{\text {air }}=340 \mathrm{~m} / \mathrm{s}$ and $\rho_{\text {air }}=1.188 \mathrm{~kg} / \mathrm{m}^{3}$. In the proposed unit cell the thickness variation of 70 to $300 \mu \mathrm{m}$ results in a phase profile which covers the full reflection phase range. Since, the mentioned thickness range is negligible compared to the total height of the unit cell $(10 \mathrm{~mm})$, the unit cells will be geometrically identical but with different reflection phases and consequently a thin and flat metasurface can be achieved. The phase profile of the proposed unit-cell versus membrane thickness is depicted in Fig. 4 for $\left(\phi=45^{\circ}, 0^{\circ}\right.$ and $\left.0<\theta<\frac{\pi}{2}\right)$ incident angles. The

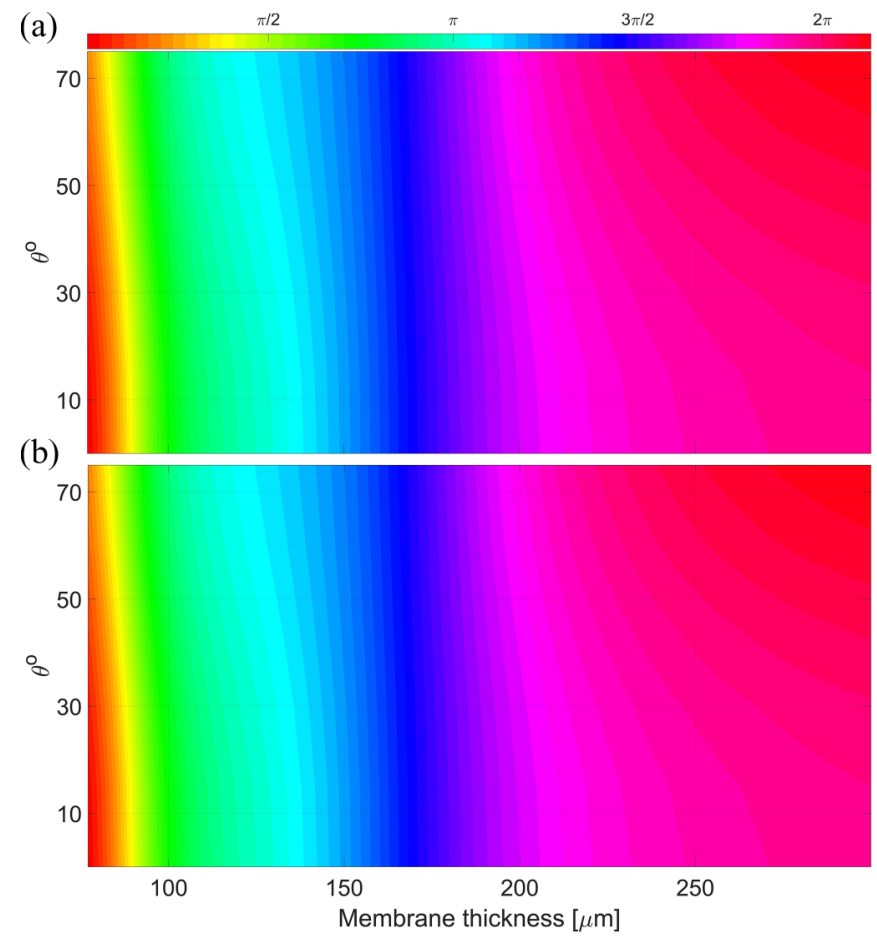

FIG. 4. Phase profiles of the proposed unit-cell $\left[\angle \Gamma\left(\theta_{i}, \phi_{i}\right)\right]$ vs membrane thickness for an acoustic pressure wave with an incident azimuth angle of (a) $\phi=0^{\circ}$ and (b) $\phi=45^{\circ}$ and elevation angle of $0^{\circ}<\theta<75^{\circ}$. close study of the unit-cell geometry and phase profile for the full study zone reveals that the dependence of the phase profile to azimuth angle $\phi$ and elevation angle $\theta$ is relatively small. This means, bearing some tolerances, the phase profile of the normal incidence can be used for the metasurface design. However, in order to achieve accurate results the phase profile of the prescribed incident direction should be retrieved.

\section{REFLECTARRAY METASURFACE}

To validate the functionality of the proposed configuration a $30 \times 30$ unit-cell reflectarray metasurface operating at $f=$ $1082 \mathrm{~Hz}$ is designed. Using such a unit cell in metasurface design, the bulky acoustic elements are replaced by the subwavelength and ultrathin ones. In the following section the acoustic reflectarray metasurface aiming at deflecting the incident acoustic wave in an anomalous way is designed and a step by step design procedure is presented.

\section{A. Design methodology}

To design a reflectarray metasurface the incident $\left(\theta_{i}, \phi_{i}\right)$ and the desired reflection $\left(\theta_{r}, \phi_{r}\right)$ angles, operating frequency, and metasurface array factors $d_{x}$ and $d_{y}$ (in this case $a$ and b) should be defined. Plugging these variables into Eq. (3) yields the surface phase function, allowing the manipulation of the reflected sound wavefront for a predefined incident direction. This surface phase function defines the required phase gradient which should be assigned to each individual unit cell with $(m, n)$ index located in $\left(m d_{x}, n d_{y}\right)$. Then, the membrane thicknesses $\left(t_{m}\right)$ are set based on this surface phase function, for the specified direction of incidence $\left(\theta_{i}, \phi_{i}\right)$.

\section{B. Numerical simulation}

Acoustic reflectarray metasurfaces are designed in three different scenarios to deflect the incident sound wave $\left(\theta_{i}, \phi_{i}\right)$ to the $\left(\theta_{r}, \phi_{r}\right)$ direction and it is simulated using Comsol. The acoustic-shell interaction physics module is used to simulated the structure, and a spherical perfectly matched layer (PML) is applied around the metasurface to truncate the simulation domain and simulate an unbounded medium. The thickness of the membranes are set to the designed values and the background pressure field is assigned to the simulation domain, representing an incident acoustic plane wave with a given incident angle. The near-field and far-field radiation pattern of the acoustic reflectarray metasurface are depicted in Fig. 5 for three distinct and predefined incident and reflected angles. Close study of the metasurface far-field radiation pattern reveals that the designed metasurfaces for predefined reflection directions of (a) $\left(\theta_{r}=45^{\circ}, \phi_{r}=120^{\circ}\right)$, (b) $\left(\theta_{r}=\right.$ $\left.31^{\circ}, \phi_{r}=212^{\circ}\right)$, and (c) $\left(\theta_{r}=31^{\circ}, \phi_{r}=30^{\circ}\right)$ deflect the sound waves to $\left(\theta_{r}=44^{\circ}, \phi_{r}=116^{\circ}\right),\left(\theta_{r}=30^{\circ}, \phi_{r}=210^{\circ}\right)$, and $\left(\theta_{r}=34^{\circ}, \phi_{r}=26^{\circ}\right)$, respectively. The small discrepancy of maximum $4^{\circ}$ between defined goals and designed values confirms the validity of both the design procedure and the unit-cell design. It is clear that the proposed metasurface is capable of handling the incoming waves with different incident angles in elevation and azimuth, and deflecting the sound wave to a predefined direction in elevation and azimuth. Moreover, investigating the far-field radiation pattern of the metasurface inset of Fig. 5 reveals that this structure has directive radiation 

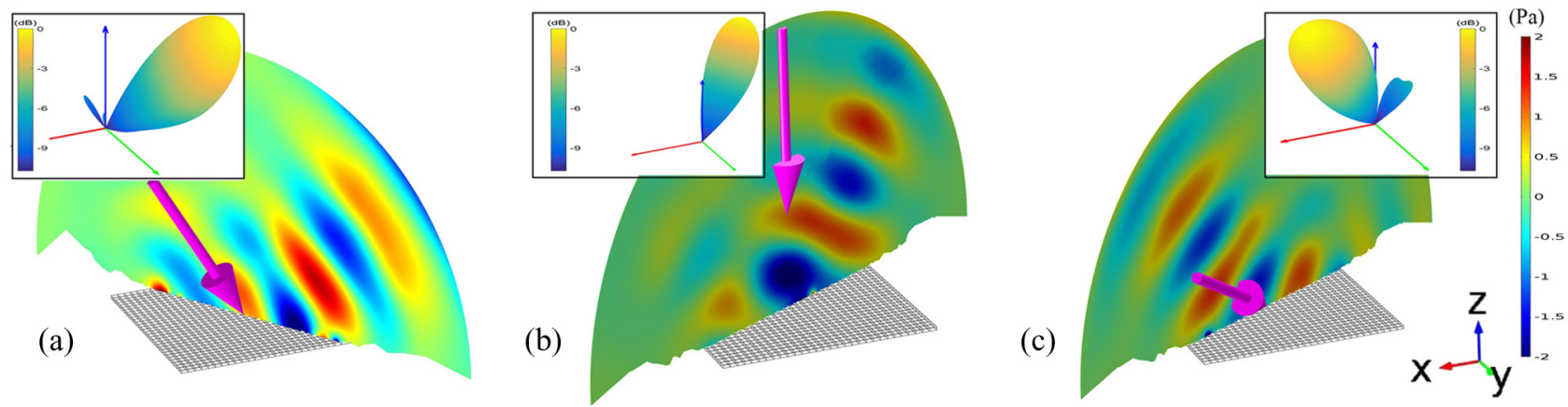

FIG. 5. The near-field scattered pressure (main) and the far-field radiation pattern (inset) for the acoustic reflectarray metasurface at $f=1082 \mathrm{~Hz}$ for three different incident and reflection angles: (a) $\left(\theta_{i}=30^{\circ}, \phi_{i}=0^{\circ}\right) \rightarrow\left(\theta_{r}=45^{\circ}, \phi_{r}=120^{\circ}\right)$, (b) $\left(\theta_{i}=0^{\circ}, \phi_{i}=\right.$ $\left.0^{\circ}\right) \rightarrow\left(\theta_{r}=30^{\circ}, \phi_{r}=210^{\circ}\right)$, and $(\mathrm{c})\left(\theta_{i}=60^{\circ}, \phi_{i}=45^{\circ}\right) \rightarrow\left(\theta_{r}=30^{\circ}, \phi_{r}=30^{\circ}\right)$. Direction of the incident acoustic wave is shown by the arrow.

which can be an alternative for acoustic reflectors. The presented acoustic metasurface is in the order of $\lambda / 30$, which is much smaller than conventional labyrinthine type structures, usually in the order of $\lambda / 3$ to $\lambda / 2$. This highlights the advantage of using membrane-based, rather than labyrinthine-type, unit cells, with a view to designing ultrathin metasurfaces.

Such design can then be employed for acoustic noise reduction/cancellation purposes, or for acoustic wave concentration for biomedical applications or even acoustic levitation. In the next section the proposed reflectarray metasurface is leveraged to design an acoustic carpet cloak.

\section{METASURFACE CARPET CLOAK}

When an acoustic wave impinges a flat surface the distribution of scattered (locally reflected) pressure field follows Snell's law. However, applying a slight modification to the surface, such as placing an object on it, the scattered field is perturbed. A target can be detected by studying the mentioned perturbed scattered field which is the principle of radars/sonars. To hide an object from an acoustic field, the scattered field distribution should be manipulated in a way that the pressure field perturbation due to the object is canceled, mimicking wave scattering from bare ground.

\section{A. Analytical description}

Such a reflectarray metasurface can be used as an acoustic carpet cloak to manipulate the scattered field from a scatterer, placed over the ground, and create the illusion of bare ground reflection. Let us suppose an acoustic scatterer is placed over an acoustically hard flat surface (bare ground). The proposed configuration is a triangular bump in the $x z$ plane which is invariant in $y$ direction (Fig. 6). If an acoustic plane wave $\left(p_{i}, k_{i}\right)$ with the incident angle $\theta$ impinges the scatterer (Fig. 6) with the slant angle $\delta$ from the ground, the reflected field locally follows Snell's law $\left(\gamma_{i}=\gamma_{r}\right)$. However, covering the scatterer with a metasurface cloak, the incident field is reflected following $\left(\gamma_{r 1}=\gamma_{i 1}+\delta\right)$ and $\left(\gamma_{r 2}=\gamma_{i 2}-\delta\right)$, in the left and the right side of the scatterer, respectively. The modification of the reflected wave angle by $\delta$ is to compensate the effect of the scatterer on the reflected wavefronts. Neglecting the diffractions from the edges, the perturbation in the scattered field due to the triangular bump is related to the local height of each point over the scatterer and this can be compensated using the reflectarray metasurface. As the reflection coefficient of an acoustic hard surface (presenting the background of the cloak) is $\Gamma=+1$, the phase discontinuity

$$
\psi\left(x_{m}\right)=-2 k h_{\mathrm{mcos}}(\theta),
$$

due to the local height of the scatterer $h_{m}$, should be compensated (Fig. 6) where $k$ is the wave number and $\theta$ is the angle of incidence with respect to the normal and $m$ is the index of surface discretization. Note that this relation is different from the electromagnetic metasurface carpet cloak over perfect electric conductor ground, where $\Gamma=-1$ and the phase gradient of the metasurface carpet cloak follows $\psi\left(x_{m}\right)=\pi-2 k h_{m} \cos (\theta)$.

\section{B. Numerical simulation}

To verify the behavior of the acoustic metasurface cloak, the geometry of Fig. 6 is numerically investigated for the cases of bare ground, bare scatterer, and cloaked scatterer exposed to the normal and slant impinging acoustic waves. The total pressure field of the isosceles triangular cloaked/uncloaked bump is studied for an acoustic incident wave using the acoustic-shell interaction module. To achieve such a design, one stack of metasurface unit cell covers a triangular bump, truncated by two parallel periodic boundary conditions in the $x z$ plane which ensures the setup is invariant in the $y$ direction. The back plane of the simulation domain representing the

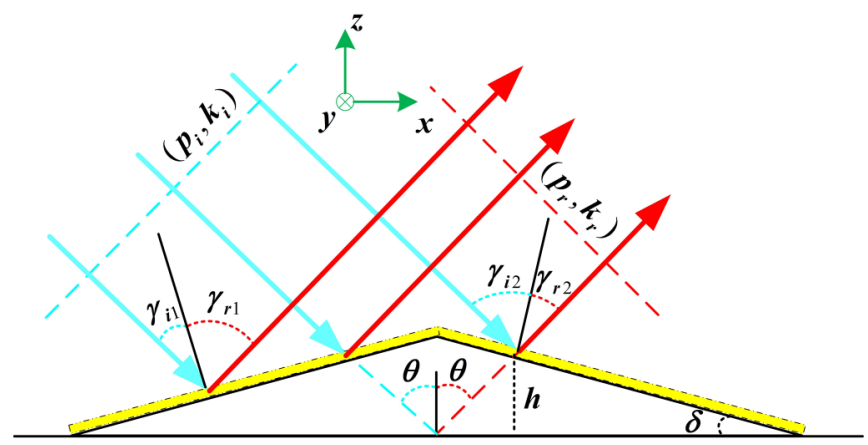

FIG. 6. A triangular bump as an acoustic scatterer (black line) cloaked with a thin acoustic metasurface (yellow). 


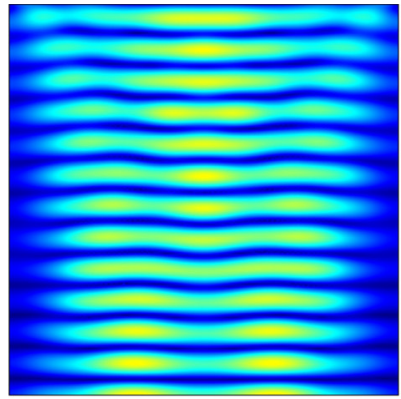

(a-i)

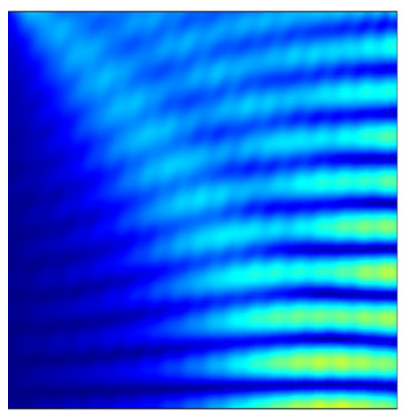

(b-i)

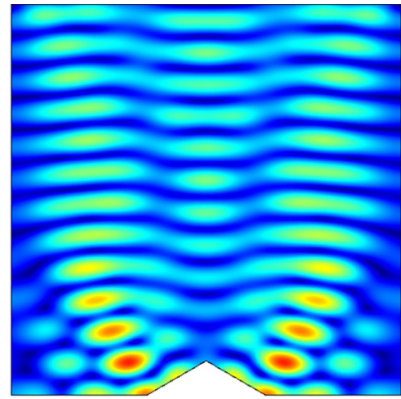

(a-ii)

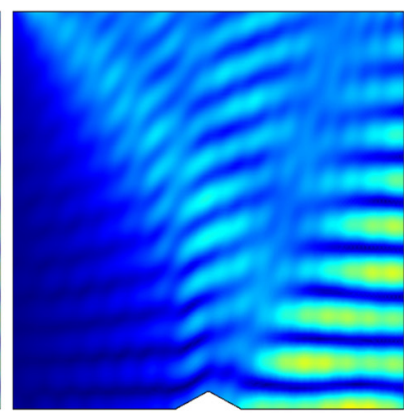

(b-ii)

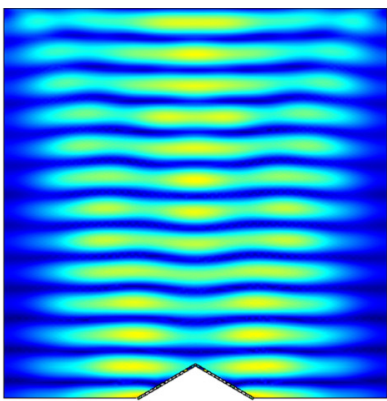

(a-iii)

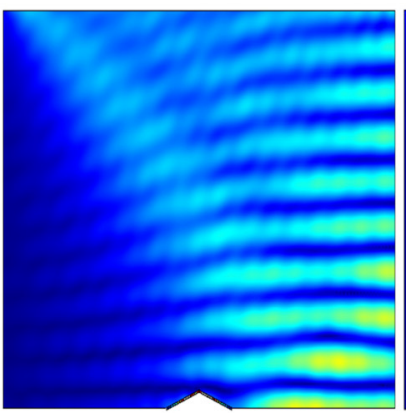

(b-iii)

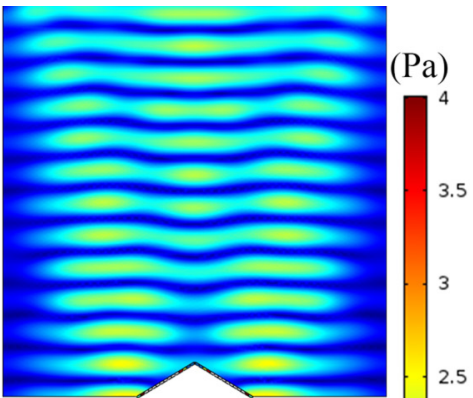

(a-iv)

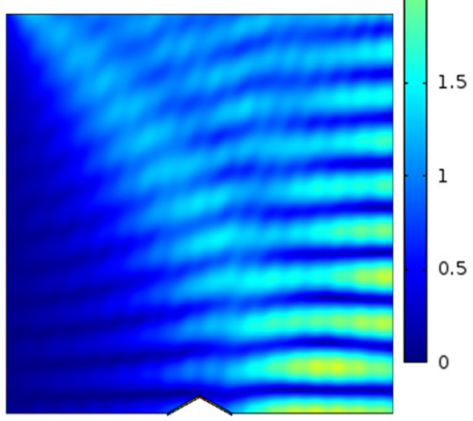

(b-iv)

FIG. 7. Absolute acoustic pressure field at $f=1082 \mathrm{~Hz}$ for (a) vertical incident and a bump with slant angle of $\delta=30^{\circ}$ and (b) $45^{\circ}$ incident angle and a bump with slant angle of $\delta=15^{\circ}$ : (i) acoustic hard surface, (ii) bare bump, (iii) cloaked bump neglecting damping, and (iv) cloaked bump including the damping $\left(\eta_{s}=0.1\right)$.

ground as well as lower surface of the metasurface representing the cavities are set to the acoustic hard boundary condition, and the upper plane of the metasurface is composed of membranes with different thickness which are defined by Eq. (8). Finally, the simulation domain is truncated by a PML semicylinder to ensure a free-space radiation condition.

The absolute acoustic pressure field is presented in Fig. 7(aii) for a triangular scatterer with the slant angle of $\delta=30^{\circ}$ which is illuminated by a normal incident acoustic plane wave. Then, this scatterer is covered with a metasurface composed of 30 membrane based unit cells and the absolute acoustic pressure field is presented in Fig. 7(a-iii), which is similar to the distribution of absolute pressure field over a bare ground depicted in Fig. 7(a-i). For the second scenario, the slant angle of the scatterer is chosen to be $\delta=15^{\circ}$ and it is covered with a 30 unit cells (the same dimension as in Sec. III) metasurface cloak and the incident angle is set to $\theta=45^{\circ}$. The absolute pressure field is compared in three different cases of scatterer with/without cloak and bare ground. Comparing the absolute pressure field over the scatterer in Fig. 7(b-ii) and over bare ground in Fig. 7(b-i) reveals that the field scattered from the uncloaked bump perturbs the absolute field and creates a shadow region. However, covering the scatterer with a thin metasurface cloak as in Fig. 7(b-iii) actually creates the illusion of the bare ground situation, showing that cloaking is also effective for slant incident angles.

Last, in order to consider the effects of viscoelastic losses, an isotropic structural loss factor $\left(\eta_{s}=0.1\right)$ is taken into account in the simulation. It should be noted that the value of structural damping does not only depend on the material properties but also on the boundary conditions and the setup configuration, and it is usually determined from measurements. However, realistic values of $\eta_{s}$ lie within the range $0.01<\eta_{s}<0.1$ for the type of elastic membranes used in the simulations [39]. Considering the upper limit of $\eta_{s}=0.1$ as a worst case, and performing the same set of simulations as in the preceding, Figs. 7(a-iv) and 7(b-iv) show that structural losses do not affect much the functionality of the carpet cloak, even with a rather high value of damping.

Comparing Figs. 7(a-iii) and 7(a-iv) (or 7(b-iii) and 7(b-iv)) shows that the effect of the damping factor is more dominant in the near field and it decreases as we go farther from the cloak. Then, the carpet cloak keeps its functionality even with introducing a high level of losses. The effect of losses has also been studied for the electromagnetic metasurface carpet cloak [8] and it was shown that, even for very large values of $\tan \delta$ (substrate loss), the cloaking metasurface is able to recover the far-field pattern. This is consistent with the results achieved on the acoustic metasurface carpet cloak proposed here, the pressure amplitudes in the far-field presenting the same color range in Figs. 7(a-iii) and 7(a-iv) (or 7(b-iii) and 7(b-iv)). A close look at the wavefronts in the top of Figs. 7(a) shows that the maximum pressure amplitude is $2.4 \mathrm{~Pa}$ (yellow color range) for the undamped case [Fig. 7(a-iii)], where it is $2.15 \mathrm{~Pa}$ (a bit greener) for the damped case [Fig. 7(a-iv)]. The level difference is then lower than $1 \mathrm{~dB}$, which is considered negligible. This result confirms that, with such membrane-based unit cells, the losses can be neglected without affecting the performance of the metasurface. Moreover, the choice of membrane-based metasurfaces does not only allow the design of ultrathin carpet cloaks, but it also overcomes the damping issues that are inherent to labyrinthine-type unit cells. 


\section{CONCLUSION}

A skinlike acoustic metasurface carpet cloak has been suggested, formulated, and numerically validated using the proposed ultrathin membrane capped cavity unit cells. The proposed cloak was leveraged to manipulate the scattered wave field in such a way to hide a target to the acoustic waves. Since the metasurface carpet cloak is easy to design and the design parameters are usually realizable in practice, it should become a good alternative to transformation based cloaks, which usually confront feasibility challenges. Moreover, this type of cloak is very versatile and can be turned into active and tunable configurations if the passive membranes are replaced by semiactive or active electroacoustic transducers [40,41].

The proposed ultrathin unit cell was also used to design an acoustic reflectarray metasurface in the audio frequency range. This very thin metasurface $(\sim \lambda / 30)$ is able to manipulate acoustic reflected waves in a prescribed direction. This may find applications in noise engineering and biomedical imaging. Furthermore, acoustic reflectarray metasurfaces may be used as an alternative to acoustic shaped reflector antennas.

To provide the mathematical tool for wavefront engineering, the generalized Snell's law for reflected acoustic waves was rederived using Fermat's principle and the wavefront of the reflected wave has been related to the phase gradient of the surface of incidence. Then, the relation between nonzero phase gradients and inhomogeneous acoustic surface impedance has been discussed.

The phase manipulation of the reflected wave using surface impedance control has led to a new design of ultrathin unit cells using membrane capped cavities. Unlike most metasurface unit cells found in the literature, where the phase manipulation relies on the effective acoustic path, here the control of the reflected wave phase lag/lead is simply achieved through surface impedance changes, thus resulting in ultrathin unit cells. Due to the high sensitivity of the unit-cell impedance to the membrane thickness, unit cells with similar geometries and dimensions but distinct impedances can be designed. Therefore, this type of unit cell is very versatile, can be used in many different designs, and will be compatible with the fast growing three-dimensional (3D) prototyping technology when the resolution of $3 \mathrm{D}$ printers will reach the micrometer scale.

\section{ACKNOWLEDGMENT}

This research was supported by the Swiss National Science Foundation under Grant No. 200020-138086.

\section{APPENDIX: MECHANICAL IMPEDANCE OF THE RECTANGULAR MEMBRANE}

To deal with lossless vibration of the clamped, isotropic, homogeneous membrane the biharmonic vibration equation

$$
\nabla^{4} \xi+\frac{k_{m}^{4}}{\omega^{2}} \frac{\partial^{2} \xi}{\partial t^{2}}=\frac{\Delta p}{D}
$$

with the following boundary conditions on the clamped edges,

$$
\begin{aligned}
\xi & =0 \\
\frac{d \xi}{d r} & =0,
\end{aligned}
$$

should be solved [35], where $\xi$ is the deformation of the plate, $D=\frac{E t_{m}^{3}}{12\left(1-v^{2}\right)}$ defined as the flexural rigidity, $\Delta p$ is the net pressure exerted on the plate, $k_{m}$ is the propagation constant and defined as $k_{m}^{2}=\omega \sqrt{\frac{\rho_{m} t_{m}}{D}}, E$ is the Young's modulus, $v$ is Poisson's ratio, $\rho_{m}$ is the mass density, and $t_{m}$ is the thickness of the plate. To find the mechanical impedance of the membrane Eq. (A1) should be solved. However, the closed-form solution for the clamped rectangular case only exists in series format [42]. Here, an indirect method is proposed to find the equivalent mass and compliance of the rectangular membrane.

If the deformation of the membrane vibrating in its first mode is modeled by [43]

$$
\xi=A\left(\cos \frac{2 \pi x}{a}-1\right)\left(\cos \frac{2 \pi y}{b}-1\right)
$$

and by neglecting the losses the energy of the vibrating plate is transformed between kinetic and potential energies:

$$
\begin{gathered}
U=\frac{1}{2} \iint D\left\{\left(\frac{\partial^{2} \xi}{\partial x^{2}}\right)^{2}+\left(\frac{\partial^{2} \xi}{\partial y^{2}}\right)^{2}+2 v\left(\frac{\partial^{2} \xi}{\partial x^{2}}\right)\left(\frac{\partial^{2} \xi}{\partial y^{2}}\right)\right. \\
\left.+2(1-v)\left(\frac{\partial^{2} \xi}{\partial x \partial y}\right)^{2}\right\} d x d y \\
K=\int \frac{\omega^{2}}{2} d m \xi^{2}
\end{gathered}
$$

where $d m=\rho_{m} d V, V$ is the membrane's volume. Using the average deformation of the membrane the kinetic and potential energy can also be described by equivalent values:

$$
\begin{gathered}
U=\frac{1}{2} \frac{\xi_{\mathrm{eq}}^{2}}{C_{e}}, \\
K=\frac{\omega^{2}}{2} m_{e} \xi_{\mathrm{eq}}^{2}
\end{gathered}
$$

where $m_{e}$ and $C_{e}$ are the equivalent mechanical mass and compliance, respectively, and $\xi_{\text {eq }}$ is the equivalent deformation of the vibrating membrane:

$$
\xi_{\text {eq }}=\frac{1}{S} \int \xi d S .
$$

By equating two relations for potential energy, Eqs. (A4) and (A6), the equivalent mechanical compliance $\left(C_{e}\right)$ is calculated. Following the same procedure for kinetic energy the equivalent mechanical mass $\left(m_{e}\right)$ is found as Eq. (5).
[1] N. Yu, P. Genevet, M. A. Kats, F. Aieta, J.-P. Tetienne, F. Capasso, and Z. Gaburro, Light propagation with phase discontinuities: generalized laws of reflection and refraction, Science 334, 333 (2011). 
[2] N. Yu and F. Capasso, Flat optics with designer metasurfaces, Nat. Mater. 13, 139 (2014).

[3] C. Argyropoulos, K. Q. Le, N. Mattiucci, G. D'Aguanno, and A. Alu, Broadband absorbers and selective emitters based on plasmonic Brewster metasurfaces, Phys. Rev. B 87, 205112 (2013).

[4] Y. Zhao and A. Alu, Manipulating light polarization with ultrathin plasmonic metasurfaces, Phys. Rev. B 84, 205428 (2011).

[5] H. T. Chen, J. F. O'Hara, A. K. Azad, A. J. Taylor, R. D. Averitt, D. B. Shrekenhamer, and W. J. Padilla, Experimental demonstration of frequency-agile terahertz metamaterials, Nature Photon. 2, 295 (2008).

[6] J. Zhang, Z. L. Mei, W. R. Zhang, F. Yang, and T. J. Cui, An ultrathin directional carpet cloak based on generalized Snell's law, Appl. Phys. Lett. 103, 151115 (2013).

[7] N. M. Estakhri and A. Alu, Ultra-thin unidirectional carpet cloak and wavefront reconstruction with graded metasurfaces, IEEE Antenn. Wireless Propag. Lett. 13, 1775 (2014).

[8] B. Orazbayev, N. M. Estakhri, M. Beruete, and A. Alu, Terahertz carpet cloak based on a ring resonator metasurface, Phys. Rev. B 91, 195444 (2015).

[9] X. Ni, Z. J. Wong, M. Mrejen, Y. Wang, and X. Zhang, An ultrathin invisibility skin cloak for visible light, Science $\mathbf{3 4 9}$, 6254 (2015).

[10] J. Zhao, B. Li, Z. N. Chen, and C.-W. Qiu, Redirection of sound waves using acoustic metasurface, Appl. Phys. Lett. 103, 151604 (2013).

[11] G. D’Aguanno, K. Q. Le, R. Trimm, A. Alù, N. Mattiucci, A. D. Mathias, N. Aközbek, and M. J. Bloemer, Broadband metamaterial for nonresonant matching of acoustic waves, Sci. Rep. 2, 340 (2012).

[12] Y.-F. Zhu, X.-Y. Zou, B. Liang, and J.-C. Cheng, Broadband unidirectional transmission of sound in unblocked channel, Appl. Phys. Lett. 106, 173508 (2015).

[13] Y.-F. Zhu, X.-Y. Zou, B. Liang, and J.-C. Cheng, Acoustic oneway open tunnel by using metasurface, Appl. Phys. Lett. 107, 113501 (2015).

[14] W. Wang, Y. Xie, A. Konneker, B.-I. Popa, and S. A. Cummer, Design and demonstration of broadband thin planar diffractive acoustic lenses, Appl. Phys. Lett. 105, 101904 (2014).

[15] J. Mei, G. Ma, M. Yang, Z. Yang, W. Wen, and P. Sheng, Dark acoustic metamaterials as super absorbers for low-frequency sound, Nat. Commun. 3, 756 (2012).

[16] G. Ma, M. Yang, S. Xiao, Z. Yang, and P. Sheng, Acoustic metasurface with hybrid resonances, Nat. Mater. 13, 873 (2014).

[17] P.-Y. Chen, M. Farhat, S. Guenneau, S. Enoch, and A. Alù, Acoustic scattering cancellation via ultrathin pseudo-surface, Appl. Phys. Lett. 99, 191913 (2011).

[18] M. Farhat, P. Y. Chen, S. Guenneau, S. Enoch, and A. Alu, Frequency-selective surface acoustic invisibility for threedimensional immersed objects, Phys. Rev. B 86, 174303 (2012).

[19] Y. Li, B. Liang, Z.-M. Gu, X.-Y. Zou, and J.-C. Cheng, Reflected wavefront manipulation based on ultrathin planar acoustic metasurfaces, Sci. Rep. 3, 2546 (2013).
[20] K. Tang, C. Qiu, M. Ke, J. Lu, Y. Ye, and Z. Liu, Anomalous refraction of airborne sound through ultrathin metasurfaces, Sci. Rep. 4, 6517 (2014).

[21] Y. Li, X. Jiang, R. Q. Li, B. Liang, X. Y. Zou, L. L. Yin, and J. C. Cheng, Experimental Realization of Full Control of Reflected Waves with Subwavelength Acoustic Metasurfaces, Phys. Rev. Appl. 2, 064002 (2014).

[22] B. Yuan, Y. Cheng, and X. Liu, Conversion of sound radiation pattern via gradient acoustic metasurface with space-coiling structure, Appl. Phys. Express 8, 027301 (2015).

[23] Z. Liang and J. Li, Extreme Acoustic Metamaterial by Coiling up Space, Phys. Rev. Lett. 108, 114301 (2012).

[24] Y. Xie, W. Wang, H. Chen, A. Konneker, B.-I. Popa, and S. A. Cummer, Wavefront modulation and subwavelength diffractive acoustics with an acoustic metasurface, Nat. Commun. 5, 5553 (2014).

[25] Y.-F. Zhu, X.-Y. Zou, R.-Q. Li, X. Jiang, J. Tu, B. Liang, and J.-C. Cheng, Dispersionless manipulation of reflected acoustic wavefront by subwavelength corrugated surface, Sci. Rep. 5, 10996 (2015).

[26] R. Al Jahdali and Y. Wu, High transmission acoustic focusing by impedance-matched acoustic meta-surfaces, Appl. Phys. Lett. 108, 031902 (2016).

[27] J. Mei and Y. Wu, Controllable transmission and total reflection through an impedance-matched acoustic metasurface, New J. Phys. 16, 123007 (2014).

[28] C. Ding, H. Chen, S. Zhai, S. Liu, and X. Zhao, The anomalous manipulation of acoustic waves based on planar metasurface with split hollow sphere, J. Phys. D 48, 045303 (2015).

[29] C. Ding, X. Zhao, H. Chen, S. Zhai, and F. Shen, Reflected wavefronts modulation with acoustic metasurface based on double-split hollow sphere, Appl. Phys. A 120, 2 (2015).

[30] Y. Li, X. Jiang, B. Liang, J. C. Cheng, and L. Zhang, MetascreenBased Acoustic Passive Phased Array, Phys. Rev. Appl. 4, 024003 (2015).

[31] C. Faure, O. Richoux, S. Félix, and V. Pagneux, Experiments on metasurface carpet cloaking for audible acoustics, Appl. Phys. Lett. 108, 6 (2016).

[32] S. Zhai, H. Chen, C. Ding, F. Shen, C. Luo, and X. Zhao, Manipulation of transmitted wavefront using ultrathin planar acoustic metasurfaces, Appl. Phys. A 120, 4 (2015).

[33] Y. Tian, Q. Wei, Y. Cheng, Z. Xu, and X. Liu, Broadband manipulation of acoustic wavefronts by pentamode metasurface, Appl. Phys. Lett. 107, 221906 (2015).

[34] J. Zhao, B. Li, Z. Chen, and C.-W. Qiu, Manipulating acoustic wavefront by inhomogeneous impedance and steerable extraordinary reflection, Sci. Rep. 3, 2537 (2013).

[35] H. Esfahlani, S. Karkar, H. Lissek, and J. R. Mosig, Acoustic dispersive prism, Sci. Rep. 6, 18911 (2016).

[36] F. Bongard, H. Lissek, and J. R. Mosig, Acoustic transmission line metamaterial with negative/zero/positive refractive index, Phys. Rev. B 82, 094306 (2010).

[37] B. I. Popa, D. Shinde, A. Konneker, and S. A. Cummer, Active acoustic metamaterials reconfigurable in real time, Phys. Rev. B 91, 220303(R) (2015).

[38] B. H. Song and J. S. Bolton, A transfer-matrix approach for estimating the characteristic impedance and wave numbers of limp and rigid porous materials, J. Acoust. Soc. Am. 107, 1131 (2000). 
[39] M. Carfagni, E. Lenzi, and M. Pierini, The loss factor as a measure of mechanical damping, in Proceedings of the 16th International Modal Analysis Conference, Santa Barbara, CA, USA (Society for Experimental Mechanics, Bethel, CT, 1998), pp. 580-584.

[40] H. Lissek, Electroacoustic metamaterials: Achieving negative acoustic properties with shunt loudspeakers, Proc. Mtgs. Acoust. 19, 030023 (2013).
[41] R. Boulandet and H. Lissek, Toward broadband electroacoustic resonators through optimized feedback control strategies, J. Sound Vib. 333, 20 (2014).

[42] J. H. Wu, A. Q. Liu, and H. L. Chen, Exact solutions for freevibration analysis of rectangular plates using Bessel functions, J. Appl. Mech 74(6), 1247 (2005).

[43] A. W. Leissa, Vibration of plates, NASA-SP-160, (1969), http://ntrs.nasa.gov/search.jsp?. 